\title{
Global Asymptotic Behavior of a Nonautonomous Competitor-Competitor-Mutualist Model
}

\author{
Shengmao Fu ${ }^{1,2}$ and Fei Qu ${ }^{1}$ \\ ${ }^{1}$ College of Mathematics and Statistics, Northwest Normal University, Lanzhou, Gansu 730070, China \\ ${ }^{2}$ School of Mathematics and Computing Science, Guilin University of Electronic Technology, Guilin, Guangxi 541004, China \\ Correspondence should be addressed to Shengmao Fu; fusm@nwnu.edu.cn
}

Received 10 May 2013; Accepted 17 July 2013

Academic Editor: Rodrigo Lopez Pouso

Copyright (C) 2013 S. Fu and F. Qu. This is an open access article distributed under the Creative Commons Attribution License, which permits unrestricted use, distribution, and reproduction in any medium, provided the original work is properly cited.

\begin{abstract}
The global asymptotic behavior of a nonautonomous competitor-competitor-mutualist model is investigated, where all the coefficients are time-dependent and asymptotically approach periodic functions, respectively. Under certain conditions, it is shown that the limit periodic system of this asymptotically periodic model admits two positive periodic solutions $\left(u_{1}^{T}, u_{2 T}, u_{3}^{T}\right),\left(u_{1 T}, u_{2}^{T}, u_{3 T}\right)$ such that $u_{i T} \leq u_{i}^{T}(i=1,2,3)$, and the sector $\left\{\left(u_{1}, u_{2}, u_{3}\right): u_{i T} \leq u_{i} \leq u_{i}^{T}, i=1,2,3\right\}$ is a global attractor of the asymptotically periodic model. In particular, we derive sufficient conditions that guarantee the existence of a positive periodic solution which is globally asymptotically stable.
\end{abstract}

\section{Introduction}

In this paper, we investigate the global asymptotic behavior of solutions for the following competitor-competitor-mutualist diffusion model:

$$
\begin{gathered}
u_{1 t}-d_{1} \Delta u_{1} \\
=g_{1} u_{1}\left(1-\frac{u_{1}}{a_{1}}-\frac{a_{2} u_{2}}{1+a_{3} u_{3}}\right) \quad \text { in } \Omega \times(0, \infty), \\
u_{2 t}-d_{2} \Delta u_{2} \\
=g_{2} u_{2}\left(1-b_{1} u_{1}-\frac{u_{2}}{b_{2}}\right) \quad \text { in } \Omega \times(0, \infty), \\
u_{3 t}-d_{3} \Delta u_{3} \\
=g_{3} u_{3}\left(1-\frac{u_{3}}{c_{1}+c_{2} u_{1}}\right) \quad \text { in } \Omega \times(0, \infty), \\
\frac{\partial u_{i}}{\partial n}=0 \quad \text { on } \partial \Omega \times(0, \infty), \quad i=1,2,3, \\
u_{i}(x, 0)=u_{i 0}(x) \quad \text { on } \Omega, i=1,2,3,
\end{gathered}
$$

where $u_{1}(x, t), u_{2}(x, t)$, and $u_{3}(x, t)$ are the densities of a mutualist-competitor, a competitor, and a mutualist population, respectively. $\Omega \subset \mathbf{R}^{N}$ is a bounded smooth domain, $\partial / \partial n$ is an outward normal derivative on $\partial \Omega$.

In 1983, Rai et al. [1] firstly presented and studied a general competitor-competitor-mutualist ordinary differential equation (ODE) model. Zheng [2] studied the problem (1)-(3) in the case where all coefficients are positive constants. He proved the local stability of the unique positive constant steady-state solution under suitable condition on the reaction rates by the method of spectral analysis for linearized operator. Xu [3] investigated the global asymptotic stability of the unique positive constant steady-state solution under some assumptions by the iteration method. Pao [4] considered the model with time delays, and, under a very simple condition on the reaction rates, proved that the time-dependent solution with any nontrivial initial function converges to the positive steady-state solution by the method of upper and lower solutions. Chen and Peng [5] proved some existence results concerning nonconstant positive steady-states for the model with cross-diffusion and demonstrated that the crossdiffusion can create patterns when the corresponding model without cross-diffusion fails. Li et al. [6] proved that this model with cross-diffusion possesses at least one coexistence 
state if cross-diffusions and cross-reactions are weak by the Schauder fixed point theory and the method of upper and lower solutions and its associated monotone iterations. Fu et al. [7] investigated the global asymptotic behavior and the global existence of time-dependent solutions for the model with cross-diffusion when the space dimension is at most 5. Very recently, Tian and Ling [8] proved that, under some conditions, a corresponding predator-prey-mutualist model with cross-diffusion admits at least a nonhomogeneous stationary solution by the stability analysis for the positive uniform solution and the Leray-Schauder degree theory and carried out numerical simulations for a Turing pattern.

For the model (1) with $T$-periodic coefficients, Tineo [9] studied the asymptotic behavior of positive solutions by the method of upper and lower solutions. Du [10] investigated the existence of positive $T$-periodic solutions by using the degree and bifurcation theories. Pao [11] proved the existence of maximal and minimal $T$-periodic solutions by the method of upper and lower solutions. Wang et al. [12] considered the local asymptotic behavior of the time-dependent solutions and the existence of periodic solutions to the model in an unbounded domain. Zhou and $\mathrm{Fu}$ [13] investigated the global asymptotic behavior of the time-dependent solutions and the existence of periodic solutions for the model with discrete delays. Very recently, replacing the usual $-\Delta u$ term by a degenerate elliptic operator as $-\Delta u^{m}$, Wang and Yin [14] proved the existence of maximal and minimal $T$-periodic solutions to the model with time delays by the Schauder fixed point theorem. It is important to note that the uniqueness of positive periodic solution is not considered in the previous references.

When $a_{3}=0$, (1) reduces to the competition diffusion system

$$
\begin{aligned}
& u_{t}-d_{1} \Delta u=u(a-b u-c v), \\
& v_{t}-d_{2} \Delta v=v(d-e u-f v),
\end{aligned}
$$

where $a=g_{1}, b=g_{1} / a_{1}, c=g_{1} / a_{2}, d=g_{2}, e=g_{2} b_{1}$, and $f=g_{2} / b_{2}$. The system (4) is a diffusion extension of the wellknown Lotka-Volterra system

$$
\begin{aligned}
& \frac{d u}{d t}=u(a-b u-c v), \\
& \frac{d v}{d t}=v(d-e u-f v) .
\end{aligned}
$$

In the case that $a, b, c, d, e$, and $f$ are positive $T$-periodic functions, the existence and asymptotic stability of periodic solutions for (5) was studied by Gopalsamy [15], Alvarez and Lazer [16], and Ahmad [17] in the 1980's. The global asymptotic behavior of (5) was studied by Ahmad and Lazer [18] and Tineo [19]. Denote $f_{L}=\inf _{x \in X} f(x)$ and $f_{M}=$ $\sup _{x \in X} f(x)$ for any function $f: X \rightarrow \mathbf{R}$. If $a, b, c, d, e$, and $f$ are positive asymptotically $T$-periodic functions on $\mathbf{R}$, Peng and Chen [20] proved that if the conditions

$$
\frac{a_{L}-\varepsilon_{0}}{d_{M}+\varepsilon_{0}}>\frac{c_{M}+\varepsilon_{0}}{f_{L}-\varepsilon_{0}}, \quad \frac{d_{L}-\varepsilon_{0}}{a_{M}+\varepsilon_{0}}>\frac{e_{M}+\varepsilon_{0}}{b_{L}-\varepsilon_{0}}
$$

are satisfied for a certain sufficiently small $\varepsilon_{0}>0$, then any positive solutions of (5) asymptotically approach the unique positive periodic solution for the limit periodic system of (5).

It is well known that periodic reaction diffusion equations are of particular interests since they can take into account seasonal fluctuations occurring in the phenomena appearing in the models, and they have been extensively studied by many researchers (see, e.g., [9-14, 19, 21]). However, so far, the research work on asymptotically periodic systems is much fewer than on the periodic ones. In fact, asymptotically periodic systems describe our world more realistically and more accurately than periodic ones to some extent. Therefore, for asymptotically periodic systems, studying the dynamics behavior is important and necessary (see, e.g., [22-27]).

In this paper, we study the global asymptotic behavior of positive solutions for the asymptotically periodic system (1). Under some conditions, it is shown that any positive solutions of the models asymptotically approach the unique strictly positive periodic solutions of the corresponding periodic system. This means that the results in Tineo [9] and the results for ODE model in Peng and Chen [20] can be extended to the asymptotically periodic reaction diffusion system and the 3species diffusion system, respectively. Furthermore, using the method of the present paper, we note that the corresponding conclusions hold for the time-dependent $n$-species LotkaVolterra systems. More specifically, we provide a way of how to use the method of upper and lower solutions to study asymptotic behavior of solutions for asymptotically periodic reaction diffusion systems. As one can see, the optimal bounds and uniqueness of positive periodic solutions will play an important role in the study of the global asymptotic behavior of periodic solutions.

\section{Permanence and Extinction}

For the sake of convenience, we introduce the two signs $\sim$ and $\prec$ for functions $u, v: \bar{\Omega} \times \mathbf{R} \rightarrow[0, \infty) \cdot u$ is said to approach $v$ asymptotically in notation, $u \sim v$, if $\lim _{t \rightarrow \infty}|u(x, t)-v(x, t)|=0$ uniformly for $x$ in $\bar{\Omega}$. Furthermore, if $\left(\varphi_{1}, \varphi_{2}, \ldots, \varphi_{n}\right)$ and $\left(\psi_{1}, \psi_{2}, \ldots, \psi_{n}\right)$ are vector functions, then $\left(\varphi_{1}, \varphi_{2}, \ldots, \varphi_{n}\right) \sim\left(\psi_{1}, \psi_{2}, \ldots, \psi_{n}\right)$ if and only if $\varphi_{i} \sim \psi_{i}(i=1,2, \ldots, n)$. We say that $u(x, t)$ is asymptotically smaller than $v(x, t)$ and write $u(x, t) \prec v(x, t)$ if $\varlimsup_{t \rightarrow \infty}(u(x, t)-v(x, t)) \leq 0$ uniformly for $x \in \bar{\Omega}$. It is clear that $u(x, t) \prec v(x, t)$ if and only, if for any $\varepsilon>0$, there exists a corresponding $t_{1}>0$ such that $u(x, t)<v(x, t)+\varepsilon$ on $\Omega \times\left[t_{1}, \infty\right)$.

Assume the following.

$\left(\mathrm{H}_{1}\right) d_{i}, A_{i}, B_{i}, C_{i}$, and $G_{i}$ are positive smooth and $T$ periodic functions on $\bar{\Omega} \times \mathbf{R}$.

$\left(\mathrm{H}_{2}\right) a_{i}, b_{i}, c_{i}$, and $g_{i}$ are positive smooth functions on $\bar{\Omega} \times \mathbf{R}$, and

$$
\left(a_{i}, b_{i}, c_{i}, g_{i}\right) \sim\left(A_{i}, B_{i}, C_{i}, G_{i}\right) .
$$


By $\left(\mathrm{H}_{2}\right)$, the limit periodic system of (1), (2) is given as follows:

$$
\begin{gathered}
u_{1 t}-d_{1} \Delta u_{1} \\
=G_{1} u_{1}\left(1-\frac{u_{1}}{A_{1}}-\frac{A_{2} u_{2}}{1+A_{3} u_{3}}\right) \quad \text { in } \Omega \times(0, \infty), \\
u_{2 t}-d_{2} \Delta u_{2} \\
=G_{2} u_{2}\left(1-B_{1} u_{1}-\frac{u_{2}}{B_{2}}\right) \quad \text { in } \Omega \times(0, \infty), \\
u_{3 t}-d_{3} \Delta u_{3} \\
=G_{3} u_{3}\left(1-\frac{u_{3}}{C_{1}+C_{2} u_{1}}\right) \quad \text { in } \Omega \times(0, \infty), \\
\frac{\partial u_{i}}{\partial n}=0 \quad \text { on } \partial \Omega \times(0, \infty)(i=1,2,3) .
\end{gathered}
$$

As a complement, we state the following main result which comes from [9, Theorem 0.3].

Theorem 1. Assume that $\left(H_{1}\right)$ holds, and

$$
\begin{gathered}
A_{2 M} B_{2 M}<1+A_{3 L} C_{1 L}, \\
A_{1 M} B_{1 M}<1 .
\end{gathered}
$$

Then (8) has the periodic solutions $\left(u_{1}^{T}, u_{2 T}, u_{3}^{T}\right)$ and $\left(u_{1 T}\right.$, $\left.u_{2}^{T}, u_{3 T}\right)$ such that $u_{i}^{T} \geq u_{i} \geq u_{i T}>0(i=1,2,3)$ for any positive T-periodic solution $\left(u_{1}, u_{2}, u_{3}\right)$ of (8). Moreover, given that $\varepsilon>0$ and a solution $\left(u_{1}, u_{2}, u_{3}\right)$ of $(8)$ with $u_{i}(x, 0) \geq$ (㓞) , there exists $t_{1}>0$ such that $u_{i T}(x, t)-\varepsilon<u_{i}(x, t)<$ $u_{i}^{T}(x, t)+\varepsilon$ on $\Omega \times\left(t_{1}, \infty\right)$.

In order to get the conditions for the permanence of (1)(3), we need to make the following optimal bounds.

Lemma 2. If (9) and (10) hold and $\left(u_{1}, u_{2}, u_{3}\right)$ is a positive smooth $T$-periodic solution of (8), then

$$
\varepsilon_{u_{i}} \leq u_{i} \leq \delta_{u_{i}} \quad(i=1,2,3)
$$

where $\delta_{u_{1}}$ is the unique positive root of $p_{1} x^{2}+q_{1} x+r_{1}=0$ and

$$
\begin{gathered}
p_{1}=A_{3 M} C_{2 M}, \\
r_{1}=A_{1 M}\left(A_{2 L} B_{2 L}-1-A_{3 M} C_{1 M}\right), \\
q_{1}=1+A_{3 M} C_{1 M}-A_{1 M} A_{3 M} C_{2 M}-A_{1 M} A_{2 L} B_{1 M} B_{2 L} .
\end{gathered}
$$

$\varepsilon_{u_{1}}$ is the unique positive root of $p_{2} x^{2}+q_{2} x+r_{2}=0$, and

$$
\begin{gathered}
p_{2}=A_{3 L} C_{2 L}, \\
r_{2}=A_{1 L}\left(A_{2 M} B_{2 M}-1-A_{3 L} C_{1 L}\right), \\
q_{2}=1+A_{3 L} C_{1 L}-A_{1 L} A_{3 L} C_{2 L}-A_{1 L} A_{2 M} B_{1 L} B_{2 M}, \\
\delta_{u_{2}}=B_{2 M}-B_{1 L} B_{2 M} \varepsilon_{u_{1}}, \\
\varepsilon_{u_{2}}=B_{2 L}-B_{1 M} B_{2 L} \delta_{u_{1}}, \\
\delta_{u_{3}}=C_{1 M}+C_{2 M} \delta_{u_{1}}, \quad \varepsilon_{u_{3}}=C_{1 L}+C_{2 L} \varepsilon_{u_{1}} .
\end{gathered}
$$

Proof. By the maximum principle (see Lemma 1.2 of [18]), we have

$$
\begin{gathered}
1-\frac{u_{1 M}}{A_{1 M}}-\frac{A_{2 L} u_{2 L}}{1+A_{3 M} u_{3 M}} \geq 0, \\
1-\frac{u_{1 L}}{A_{1 L}}-\frac{A_{2 M} u_{2 M}}{1+A_{3 L} u_{3 L}} \leq 0, \\
1-B_{1 L} u_{1 L}-\frac{u_{2 M}}{B_{2 M}} \geq 0, \\
1-B_{1 M} u_{1 M}-\frac{u_{2 L}}{B_{2 L}} \leq 0, \\
1-\frac{u_{3 M}}{C_{1 M}+C_{2 M} u_{1 M}} \geq 0, \\
1-\frac{u_{3 L}}{C_{1 L}+C_{2 L} u_{1 L}} \leq 0 .
\end{gathered}
$$

Hence, $p_{1} u_{1 M}^{2}+q_{1} u_{1 M}+r_{1} \leq 0$. Since $p_{1}>0$ and $r_{1}<0$ (by (9)), we can see immediately that $u_{1 M} \leq \delta_{u_{1}}$. Similarly, if $\varepsilon_{u_{1}}$ is the unique positive root of $p_{2} x^{2}+q_{2} x+r_{2}=0$, then $u_{1 L} \geq \varepsilon_{u_{1}}$. So,

$$
\begin{aligned}
& \varepsilon_{u_{2}} \leq u_{2 L} \leq u_{2 M} \leq \delta_{u_{2}}, \\
& \varepsilon_{u_{3}} \leq u_{3 L} \leq u_{3 M} \leq \delta_{u_{3}} .
\end{aligned}
$$

Evidently, $\varepsilon_{u_{3}}>0$. By (9) and (10), we have

$$
\frac{p_{1}}{B_{1 M}^{2}}+\frac{q_{1}}{B_{1 M}}+r_{1}>0
$$

from which it follows that $\varepsilon_{u_{2}}>0$. This completes the proof.

Corollary 3. Assume that (9) and (10) hold. If $A_{i}, B_{i}$, and $C_{i}$ are positive constants, then (9) has the unique positive periodic solution $\left(r, B_{2}\left(1-B_{1} r\right), C_{1}+C_{2} r\right)$, where $r$ is the unique positive root of $p_{1} x^{2}+q_{1} x+r_{1}=0$. 
The main results in this section are the following theorems.

Theorem 4 (permanence). Assume that $\left(H_{1}\right),\left(H_{2}\right),(9)$, and (10) hold. Then (8) has the positive T-periodic solutions $\left(u_{1 T}, u_{2}^{T}, u_{3 T}\right)$ and $\left(u_{1}^{T}, u_{2 T}, u_{3}^{T}\right)$ such that $u_{i T} \leq u_{i}^{T}(i=$ $1,2,3)$. Moreover, if $\left(u_{1}, u_{2}, u_{3}\right)$ is the solution of (1)-(3) with smooth initial values $u_{i 0}(x) \geq(\not \equiv) 0$, then

$$
u_{i T} \prec u_{i} \prec u_{i}^{T} \quad(i=1,2,3)
$$

Remark 5. Under the assumptions of Theorem 4, the system (1), (2) is permanent, the sector $\left\langle u_{T}, u^{T}\right\rangle=\{u \in C(\bar{\Omega} \times \mathbf{R})$ : $\left.u_{T} \leq u \leq u^{T}\right\}$ is a global periodic attractor of (1), (2), and its trivial and semitrivial periodic solutions are unstable. Furthermore, if $A_{i}, B_{i}$, and $C_{i}$ are positive constants, then $\left(r, B_{2}\left(1-B_{1} r\right), C_{1}+C_{2} r\right)$ is the unique globally asymptotically stable solution of (8).

Theorem 6. Assume that $\left(H_{1}\right)$ and $\left(H_{2}\right)$ hold. Then one has the following conclusions.

(1) (Extinction of $u_{2}$ ) Assume that (9) holds and that $A_{1 L} B_{1 L} \geq 1$. Then (8) has a T-periodic solution $\left(U_{1}, U_{2}, U_{3}\right)$ such that $U_{1}>0, U_{2}=0, U_{3}>0$, and

$$
\lim _{t \rightarrow \infty}\left|u_{i}(x, t)-U_{i}(x, t)\right|=0 \quad(i=1,2,3)
$$

uniformly on $\bar{\Omega}$, for any positive solution $\left(u_{1}, u_{2}, u_{3}\right)$ of (1)-(3).

(2) (Extinction of $u_{1}$ ) Assume that (10) holds and that

$$
A_{2 L} B_{2 L} \geq 1+A_{3 M} C_{1 M}+A_{3 M} A_{1 M} C_{2 M}
$$

Then (8) has a T-periodic solution $\left(U_{1}, U_{2}, U_{3}\right)$ with $U_{1}=0, U_{2}>0$, and $U_{3}>0$ satisfying (18), where $\left(u_{1}, u_{2}, u_{3}\right)$ is any positive solution of (1)-(3).

Proof of Theorem 4. By (9) and (10), there exists a sufficiently small $\varepsilon_{0}>0$ such that, for $\delta \in\left(0, \varepsilon_{0}\right)$,

$$
\begin{gathered}
{\left[\frac{\left(G_{1}+\delta\right)\left(A_{2}+\delta\right)}{G_{1}-\delta}\right]_{M}\left[\frac{\left(G_{2}+\delta\right)\left(B_{2}+\delta\right)}{G_{2}-\delta}\right]_{M}} \\
<1+\left(A_{3 L}-\delta\right)\left[\frac{\left(G_{3}-\delta\right)\left(C_{1}-\delta\right)}{G_{3}+\delta}\right]_{L} \\
{\left[\frac{\left(G_{1}+\delta\right)\left(A_{1}+\delta\right)}{G_{1}-\delta}\right]_{M}\left[\frac{\left(G_{2}+\delta\right)\left(B_{1}+\delta\right)}{G_{2}-\delta}\right]_{M}<1 .}
\end{gathered}
$$

Consider two auxiliary systems as follows:

$$
\begin{aligned}
& u_{1 t}-d_{1} \Delta u_{1} \\
& =u_{1}\left[\left(G_{1}+\delta\right)-\frac{\left(G_{1}-\delta\right) u_{1}}{A_{1}+\delta}\right. \\
& \left.-\frac{\left(G_{1}-\delta\right)\left(A_{2}-\delta\right) u_{2}}{1+\left(A_{3}+\delta\right) u_{3}}\right] \quad \text { in } \Omega \times(0, \infty), \\
& u_{2 t}-d_{2} \Delta u_{2} \\
& =u_{2}\left[\left(G_{2}-\delta\right)-\left(G_{2}+\delta\right)\left(B_{1}+\delta\right) u_{1}\right. \\
& \left.-\frac{\left(G_{2}+\delta\right) u_{2}}{B_{2}-\delta}\right] \quad \text { in } \Omega \times(0, \infty), \\
& =u_{3}\left[\left(G_{3}+\delta\right)\right. \\
& \left.-\frac{\left(G_{3}-\delta\right) u_{3}}{\left(C_{1}+\delta\right)+\left(C_{2}+\delta\right) u_{1}}\right] \quad \text { in } \Omega \times(0, \infty), \\
& \frac{\partial u_{i}}{\partial n}=0 \quad \text { on } \partial \Omega \times(0, \infty)(i=1,2,3) \text {, }
\end{aligned}
$$

$$
\begin{aligned}
& u_{1 t}-d_{1} \Delta u_{1} \\
& =u_{1}\left[\left(G_{1}-\delta\right)-\frac{\left(G_{1}+\delta\right) u_{1}}{A_{1}-\delta}\right. \\
& \left.-\frac{\left(G_{1}+\delta\right)\left(A_{2}+\delta\right) u_{2}}{1+\left(A_{3}-\delta\right) u_{3}}\right] \quad \text { in } \Omega \times(0, \infty) \text {, } \\
& u_{2 t}-d_{2} \Delta u_{2} \\
& =u_{2}\left[\left(G_{2}+\delta\right)-\left(G_{2}-\delta\right)\left(B_{1}-\delta\right) u_{1}\right. \\
& \left.-\frac{\left(G_{2}-\delta\right) u_{2}}{B_{2}+\delta}\right] \quad \text { in } \Omega \times(0, \infty), \\
& u_{3 t}-d_{3} \Delta u_{3} \\
& =u_{3}\left[\left(G_{3}-\delta\right)-\frac{\left(G_{3}+\delta\right) u_{3}}{\left(C_{1}-\delta\right)+\left(C_{2}-\delta\right) u_{1}}\right] \\
& \text { in } \Omega \times(0, \infty) \text {, } \\
& \frac{\partial u_{i}}{\partial n}=0 \quad \text { on } \partial \Omega \times(0, \infty)(i=1,2,3) .
\end{aligned}
$$

By (20), (21), and Theorem 1, (22) has the positive $T$ periodic solutions $\left(U_{1 \delta}, u_{2}^{\delta}, U_{3 \delta}\right)$ and $\left(U_{1}^{\delta}, u_{2 \delta}, U_{3}^{\delta}\right)$ such that $U_{i \delta} \leq u_{i} \leq U_{i}^{\delta}(i=1,3)$ and $u_{2 \delta} \leq u_{2} \leq u_{2}^{\delta}$, for any positive $T$-periodic solution $\left(u_{1}, u_{2}, u_{3}\right)$ of $(22)$. Moreover, if 
$\left(u_{1}, u_{2}, u_{3}\right)$ is a solution of (22) with nontrivial nonnegative initial values, then, for any $\varepsilon>0$, there exists $t_{\varepsilon}>0$ such that

$$
\begin{gathered}
U_{i \delta}(x, t)-\varepsilon<u_{i}(x, t)<U_{i}^{\delta}(x, t)+\varepsilon \quad(i=1,3), \\
u_{2 \delta}(x, t)-\varepsilon<u_{2}(x, t)<u_{2}^{\delta}(x, t)+\varepsilon,
\end{gathered}
$$

for all $x \in \bar{\Omega}$ and $t>t_{\varepsilon}$. Similarly, (23) has the positive $T$ periodic solutions $\left(u_{1 \delta}, U_{2}^{\delta}, u_{3 \delta}\right)$ and $\left(u_{1}^{\delta}, U_{2 \delta}, u_{3}^{\delta}\right)$ such that $u_{i \delta} \leq u_{i} \leq u_{i}^{\delta}(i=1,3)$ and $U_{2 \delta} \leq u_{2} \leq U_{2}^{\delta}$, for any positive $T$-periodic solution $\left(u_{1}, u_{2}, u_{3}\right)$ of (23). Furthermore, if $\left(u_{1}, u_{2}, u_{3}\right)$ is a solution of (23) with nontrivial nonnegative initial values, then, for the previous $\varepsilon>0$, there exists $t_{\varepsilon}^{\prime}>0$ such that, for all $x \in \bar{\Omega}$ and $t>t_{\varepsilon}^{\prime}$,

$$
\begin{gathered}
u_{i \delta}(x, t)-\varepsilon<u_{i}(x, t)<u_{i}^{\delta}(x, t)+\varepsilon \quad(i=1,3), \\
U_{2 \delta}(x, t)-\varepsilon<u_{2}(x, t)<U_{2}^{\delta}(x, t)+\varepsilon .
\end{gathered}
$$

Now we prove

$$
u_{i \delta} \leq u_{i T} \leq U_{i \delta}, \quad u_{i}^{\delta} \leq u_{i}^{T} \leq U_{i}^{\delta}
$$

where $\left(u_{1 T}, u_{2}^{T}, u_{3 T}\right)$ and $\left(u_{1}^{T}, u_{2 T}, u_{3}^{T}\right)$ are positive $T$-periodic solutions of $(8)$ (see Theorem 1$)$. Let $\left(U_{1}, u_{2}, U_{3}\right),\left(p_{1}, p_{2}, p_{3}\right)$, and $\left(u_{1}, U_{2}, u_{3}\right)$ be the solutions of $(22),(8)$, and (23), respectively, which all satisfy the same initial conditions. It is easily testified that $\left(U_{1}, U_{2}, U_{3}\right),\left(u_{1}, u_{2}, u_{3}\right)$ are the upper and lower solutions of (8) and (3), respectively. So from [28, Corollary 5.2.10], we see that

$$
u_{i} \leq p_{i} \leq U_{i} \quad(i=1,2,3) .
$$

For sufficiently small $m>0$ and $\delta>0$, define

$$
\begin{gathered}
r_{1}=\left[\frac{\left(G_{1}+\delta\right)\left(A_{1}+\delta\right)}{G_{1}-\delta}\right]_{M}, \\
r_{2}=\left[\frac{\left(G_{2}+\delta\right)\left(B_{2}+\delta\right)}{G_{2}-\delta}\right]_{M} \\
r_{3}=\left[\frac{\left(G_{3}+\delta\right)\left(C_{1}+\delta\right)}{G_{3}-\delta}\right]_{M}+\left[\frac{\left(G_{3}+\delta\right)\left(C_{2}+\delta\right)}{G_{3}-\delta}\right]_{M} r_{1}, \\
s_{0}=\left[\frac{\left(G_{3}-\delta\right)\left(C_{1}-\delta\right)}{G_{3}+\delta}\right]_{L}+\left[\frac{\left(G_{3}-\delta\right)\left(C_{2}-\delta\right)}{G_{3}+\delta}\right]_{L} m .
\end{gathered}
$$

Choose $\left(u_{10}(x), u_{20}(x), u_{30}\right)=\left(r_{1}, m, r_{2}\right)$. Then $\left(r_{1}, r_{2}, r_{3}\right)$ and $(m, m, s)$ are the ordered upper and lower solutions of (22) and (3) (also of (23) and (3) and of (8) and (3)). Applying the same technique from [18, Theorem 4.1], we can prove that the solution $\left(U_{1}, u_{2}, U_{3}\right)$ of (22) and (3), the solution $\left(u_{1}, U_{2}, u_{3}\right)$ of $(23)$ and (3), and the solution $\left(p_{1}, p_{2}, p_{3}\right)$ of $(8)$ and (3) satisfy, respectively,

$$
\begin{aligned}
\lim _{n \rightarrow \infty}\left(U_{1}(x, t+n T), u_{2}(x, t+n T), U_{3}(x, t+n t)\right) \\
\quad=\left(U_{1}^{\delta}(x, t), u_{2 \delta}(x, t), U_{3}^{\delta}(x, t)\right), \\
\lim _{n \rightarrow \infty}\left(u_{1}(x, t+n t), U_{2}(x, t+n T), u_{3}(x, t+n T)\right) \\
\quad=\left(u_{1}^{\delta}(x, t), U_{2 \delta}(x, t), u_{3}^{\delta}(x, t)\right), \\
\lim _{n \rightarrow \infty}\left(p_{1}(x, t+n T), p_{2}(x, t+n T), p_{3}(x, t+n T)\right) \\
=\left(u_{1}^{T}(x, t), u_{2 T}(x, t), u_{3}^{T}(x, t)\right) .
\end{aligned}
$$

It follows from (27) that $u_{i}^{\delta} \leq u_{i}^{T} \leq U_{i}^{\delta}(i=1,3)$ and that $u_{2 \delta} \leq u_{2 T} \leq U_{2 \delta}$. Similarly, choose $\left(u_{10}(x), u_{20}(x), u_{30}(x)\right)=$ $\left(m, r_{2}, s\right)$; we can prove the other inequalities of (26).

Denote by $\varepsilon_{1 u_{i}}, \delta_{1 u_{i}}, \varepsilon_{2 u_{i}}$, and $\delta_{2 u_{i}}$ the optimal bounds for the positive periodic solutions of problems (22) and (23), respectively, (see Lemma 2). Then

$$
\begin{gathered}
\delta_{2 u_{i}} \leq \delta_{u_{i}} \leq \delta_{1 u_{i}}, \\
\varepsilon_{2 u_{i}} \leq \varepsilon_{u_{i}} \leq \varepsilon_{1 u_{i}} \quad(i=1,3), \\
\delta_{1 u_{2}} \leq \delta_{u_{2}} \leq \delta_{2 u_{2}}, \quad \varepsilon_{1 u_{2}} \leq \varepsilon_{u_{2}} \leq \varepsilon_{2 u_{2}} .
\end{gathered}
$$

Moreover,

$$
\begin{gathered}
\varepsilon_{2 u_{i}} \leq u_{i \delta} \leq U_{i}^{\delta} \leq \delta_{1 u_{i}} \quad(i=1,3), \\
\varepsilon_{1 u_{2}} \leq u_{2 \delta} \leq U_{2}^{\delta} \leq \delta_{2 u_{2}} \\
u_{i \delta_{1}}<u_{i \delta_{2}}, \quad U_{i}^{\delta_{1}}>U_{i}^{\delta_{2}}
\end{gathered}
$$

for $0<\delta_{2}<\delta_{1}<\varepsilon_{0}$. By using the dominated convergence theorem and the bootstrap arguments (see [18]), we have

$$
\begin{aligned}
& \lim _{\delta \rightarrow 0+}\left(U_{1}^{\delta}, u_{2 \delta}, U_{3}^{\delta}\right)=\left(u_{1}^{s}, u_{2 s}, u_{3}^{s}\right), \\
& \lim _{\delta \rightarrow 0+}\left(u_{1 \delta}, U_{2}^{\delta}, u_{3 \delta}\right)=\left(u_{1 s}, u_{2}^{s}, u_{3 s}\right)
\end{aligned}
$$

uniformly for $(x, t)$ on $\bar{\Omega} \times \mathbf{R}$, and $\left(u_{1 s}, u_{2}^{s}, u_{3 s}\right)$ and $\left(u_{1}^{s}, u_{2 s}, u_{3}^{s}\right)$ are the positive $T$-periodic solutions of (8).

From Theorem 1, we see that $u_{i T} \leq u_{i s}$ and $u_{i}^{s} \leq u_{i}^{T}(i=$ $1,2,3)$. It follows from (26) and (32) that $u_{i s} \leq u_{i T}, u_{i}^{T} \leq$ $u_{i}^{s}(i=1,2,3)$. So,

$$
u_{i}^{s}=u_{i}^{T}, \quad u_{i s}=u_{i T} \quad(i=1,2,3) .
$$

Therefore, given that $\varepsilon>0$, by (32) and (33), there exists $\delta_{0} \in\left(0, \varepsilon_{0}\right)$ such that

$$
u_{i T}-\frac{\varepsilon}{2}<u_{i \delta_{0}} \leq U_{i}^{\delta_{0}}<u_{i}^{T}+\frac{\varepsilon}{2} \quad(i=1,2,3) .
$$

Since $\left(a_{i}, b_{i}, c_{i}, g_{i}\right) \sim\left(A_{i}, B_{i}, C_{i}, G_{i}\right)$, for the previous $\delta_{0}$, there exists $T_{\delta_{0}}>0$ such that, for $t>T_{\delta_{0}}$,

$$
A_{i}-\delta_{0}<a_{i}<A_{i}+\delta_{0}, \ldots, G_{i}-\delta_{0}<g_{i}<G_{i}+\delta_{0} .
$$


Denote by $\left(u_{1}, u_{2}, u_{3}\right)$ the solution of (1)-(3), and denote by $\left(u_{1}^{*}, u_{2 *}, u_{3}^{*}\right),\left(U_{1}, U_{2}, U_{3}\right)$, and $\left(u_{1 *}, u_{2}^{*}, u_{3 *}\right)$ the solutions of problems (22), (8), and (23), respectively, which all satisfy the same initial conditions $u_{i}\left(x, T_{\delta_{0}}+1\right)=u_{i 0}(x) \geq(\not \equiv) 0(i=$ $1,2,3)$. Analogous to (27), we have $u_{i *} \leq u_{i} \leq u_{i}^{*}(i=1,2,3)$ for $t>T_{\delta_{0}}$. By (24), there exists $T_{1}>T_{\delta_{0}}+1$ such that

$$
\begin{gathered}
U_{i \delta_{0}}-\frac{\varepsilon}{2}<u_{i}^{*}<U_{i}^{\delta_{0}}+\frac{\varepsilon}{2} \quad(i=1,3), \\
u_{2 \delta_{0}}-\frac{\varepsilon}{2}<u_{2 *}<u_{2}^{\delta_{0}}+\frac{\varepsilon}{2}
\end{gathered}
$$

for $t>T_{1}$. Similarly, by (25), there exists $T_{2}>T_{\delta_{0}}+1$ such that

$$
\begin{gathered}
u_{i \delta_{0}}-\frac{\varepsilon}{2}<u_{i *}<u_{i}^{\delta_{0}}+\frac{\varepsilon}{2} \quad(i=1,3), \\
U_{2 \delta_{0}}-\frac{\varepsilon}{2}<u_{2}^{*}<U_{2}^{\delta_{0}}+\frac{\varepsilon}{2}
\end{gathered}
$$

for $t>T_{2}$. Hence, by (34)-(37), we have

$$
u_{i T}-\varepsilon<u_{i *} \leq u_{i} \leq u_{i}^{*}<u_{i}^{T}+\varepsilon
$$

for $t>\max \left\{T_{1}, T_{2}\right\}$. This completes the proof.

\section{Global Stability}

In order to get conditions of global stability for (1)-(3), we need the following result.

Lemma 7. Let $(u, v, w)$ be a T-periodic solution for the linear problem

$$
\begin{gathered}
u_{t}-d_{1} \Delta u-\Sigma a_{i} u_{x_{i}}=M_{1}(-A u+B v-C w), \\
v_{t}-d_{2} \Delta v-\Sigma b_{i} v_{x_{i}}=M_{2}(D u-E v), \\
w_{t}-d_{3} \Delta w-\Sigma c_{i} w_{x_{i}}=M_{3}(F u-G w), \\
\frac{\partial u}{\partial n}=\frac{\partial v}{\partial n}=\frac{\partial w}{\partial n}=0 \quad \text { on } \partial \Omega \times \mathbf{R},
\end{gathered}
$$

where $d_{i}, M_{i}, A, B, C, D, E, F$, and $G$ are positive smooth $T$ periodic functions on $\bar{\Omega} \times \mathbf{R}$ and where $a_{i}, b_{i}$, and $c_{i}$ are smooth T-periodic functions. If

$$
B\left(\frac{D}{E}\right)_{M}+C\left(\frac{F}{G}\right)_{M}<A
$$

then $u=v=w=0$.

Proof. Let $(u, v, w)$ be a smooth $T$-periodic solution of (39), and let positive constants $\varepsilon, m, k$, and $l$ be chosen so that

$$
\begin{gathered}
M_{1 L}(A-B k-C l) \geq \varepsilon, \quad M_{2 L}(E k-D) \geq \varepsilon k \\
M_{3 L}(G l-F) \geq \varepsilon l, \quad u_{M} \leq m, \\
v_{M} \leq m k, \quad w_{M} \leq m l .
\end{gathered}
$$

Such choices are obviously possible because (40) holds.
It is easy to verify that $m(1, k, l) e^{-\varepsilon t}$ and $-m(1, k, l) e^{-\varepsilon t}$ are a pair of ordered upper and lower solutions of (39). Thus, $-m e^{-\varepsilon t} \leq u(x, t) \leq m e^{-\varepsilon t}$. This implies that $u(x, t)=0$ because $u(x, t)$ is $T$-periodic in $t$.

Similarly, $v=0=w$. This completes the proof.

Lemma 8 (uniqueness). Assume that (9) and (10) hold. If

$$
\begin{gathered}
\frac{B_{1 M} B_{2 M}}{\varepsilon_{u_{2}}}+\frac{A_{3 M} C_{2 M} \delta_{u_{3}}^{2}}{\left(1+A_{3 L} \varepsilon_{u_{3}}\right) \varepsilon_{u_{3}}^{2}} \\
<\frac{\varepsilon_{u_{1}}\left(1+A_{3 L} \varepsilon_{u_{3}}\right)}{A_{1 M} A_{2 M} \delta_{u_{1}} \delta_{u_{2}}}
\end{gathered}
$$

then the problem (8) has a unique positive T-periodic solution.

Proof. Let $\alpha=u_{1}^{T} / u_{1 T}-1, \beta=1-u_{2 T} / u_{2}^{T}$ and $\gamma=u_{3}^{T} / u_{3 T}-1$. By Theorem 1, we have

$$
\begin{gathered}
\alpha_{t}-d_{1} \Delta \alpha-\frac{2 d_{1}}{U_{1 T}} \sum u_{1 T x_{i}} \alpha_{x_{i}} \\
=\frac{g_{1} u_{1}^{T}}{u_{1 T}}\left[\frac{-u_{1 T} \alpha}{A_{1}}+\frac{A_{2} u_{2}^{T} \beta}{1+A_{3} u_{3}^{T}}\right. \\
\left.-\frac{A_{2} A_{3} u_{2}^{T} u_{3 T} \gamma}{\left(1+A_{3} u_{3}^{T}\right)\left(1+A_{3} u_{3 T}\right)}\right], \\
\beta_{t}-d_{2} \Delta \beta-\frac{2 d_{2}}{U_{2}^{T}} \sum u_{2 x_{i}}^{T} \beta_{x_{i}} \\
=\frac{g_{2} u_{2 T}}{u_{2}^{T}}\left[B_{1} u_{1 T} \alpha-\frac{u_{2}^{T} \beta}{B_{2}}\right], \\
\gamma_{t}-d_{3} \Delta \gamma-\frac{2 d_{3}}{U_{3 T}} \sum u_{3 T x_{i}} \gamma_{x_{i}} \\
=\frac{\partial \alpha}{\left(C_{1}+C_{2} u_{1 T}\right) u_{3 T}}\left[\frac{\partial \beta}{\partial n}=\frac{\partial \gamma}{\partial n}=0 \quad \text { on } \partial \Omega \times(0, \infty) .\right. \\
\left.C_{1}+C_{2} u_{1}^{T}-u_{3 T} \gamma\right],
\end{gathered}
$$

It follows from Lemmas 2 and 7 and the conditions (9), (10), and (42) that $\alpha=\beta=\gamma=0$. This completes the proof.

Theorem 9. If all conditions of Theorem 4 and (42) are satisfied, then

$$
\left(u_{1 T}, u_{2}^{T}, u_{3 T}\right)=\left(u_{1}^{T}, u_{2 T}, u_{3}^{T}\right) \sim\left(u_{1}, u_{2}, u_{3}\right)
$$

for any positive solution $\left(u_{1}, u_{2}, u_{3}\right)$ of (1)-(3).

Proof. By some elementary calculations, we know that Theorem 9 is an immediate corollary of Theorem 4 and Lemma 8. 
Example 10. Consider the following asymptotically periodic system:

$$
\begin{gathered}
u_{1 t}-(2+\sin t) u_{1 x x} \\
=\left(1+\sin ^{2}(t+x) e^{-t^{2}}\right) u_{1}\left(1-u_{1}-\frac{u_{2}}{1+u_{3}}\right), \\
u_{2 t}-(2-\sin t) u_{2 x x} \\
=u_{2}\left(1-\left(\frac{3}{8}+\frac{1}{24} \cos ^{2}(t+x)\right) u_{1}-u_{2}\right) \quad \text { in }(0,1) \times \mathbf{R}, \\
u_{3 t}-u_{3 x x} \\
\quad=u_{3}\left(1-\frac{u_{3}}{5 / 12+(7 / 12) \sin ^{2}(t-x)+u_{1}}\right), \\
u_{i x}(0, t)=u_{i x}(1, t)=0 \text { on } \mathbf{R}(i=1,2,3) .
\end{gathered}
$$

It is not hard to verify that all conditions of Theorem 9 are satisfies. Thus, any positive solution of (45) asymptotically approach the unique positive periodic solution of the limit periodic system of (45).

\section{Case $a_{3}=0$}

The following results are natural generalizations of the main results in [20] which can be proved in the similar way as to prove Theorems 4 and 9.

\section{Theorem 11. Assume the following.}

$\left(\mathrm{A}_{1}\right) d_{1}, d_{2}, A, B, C, D, E$, and $F$ are positive smooth $T$ periodic functions on $\bar{\Omega} \times \mathbf{R}$.

$\left(\mathrm{A}_{2}\right) a, b, c, d, e$, and $f$ are positive smooth functions on $\bar{\Omega} \times$ R:

$$
\begin{gathered}
(a, b, c, d, e, f) \sim(A, B, C, D, E, F) \\
\left(\frac{F}{D}\right)_{L}>\left(\frac{C}{A}\right)_{M}, \quad\left(\frac{B}{A}\right)_{L}>\left(\frac{E}{D}\right)_{M} .
\end{gathered}
$$

Then the limit periodic system of (4)

$$
\begin{gathered}
U_{t}-k_{1} \Delta U=U(A-B U-C V) \quad \text { in } \Omega \times \mathbf{R}, \\
V_{t}-k_{2} \Delta V=V(D-E U-F V) \quad \text { in } \Omega \times \mathbf{R}, \\
\frac{\partial U}{\partial n}=0=\frac{\partial V}{\partial n} \quad \text { on } \partial \Omega \times \mathbf{R}
\end{gathered}
$$

has the positive T-periodic maxmini solution $\left(U^{T}, V_{T}\right)$ and minimax solution $\left(U_{T}, V^{T}\right)$. Moreover, if $(u, v)$ is any positive solution of (4) with smooth initial value $\left(u_{0}, v_{0}\right)$, then $U_{T} \prec$ $u \prec U^{T}$ and $V_{T} \prec v \prec V^{T}$. In addition, if

$$
\begin{aligned}
\left(\frac{C}{A}\right)_{M} & \left(\frac{E}{D}\right)_{M}\left[\left(\frac{F}{D}\right)_{M}-\left(\frac{C}{A}\right)_{L}\right]\left[\left(\frac{B}{A}\right)_{M}-\left(\frac{E}{D}\right)_{L}\right] \\
< & \left(\frac{B}{A}\right)_{L}\left(\frac{F}{D}\right)_{L}\left[\left(\frac{F}{D}\right)_{L}-\left(\frac{C}{A}\right)_{M}\right]\left[\left(\frac{B}{A}\right)_{L}-\left(\frac{E}{D}\right)_{M}\right],
\end{aligned}
$$

then (47) has the unique positive T-periodic solution $(U, V)$ and

$$
(u(\cdot, t), v(\cdot, t)) \sim(U(\cdot, t), V(\cdot, t)) .
$$

\section{Acknowledgments}

The authors would like to thank the referees for their helpful comments. This work is supported by the China National Natural Science Foundation (Grants nos. 11061031 and 11261053) and the Fundamental Research Funds for the Gansu University.

\section{References}

[1] B. Rai, H. I. Freedman, and J. F. Addicott, "Analysis of threespecies models of mutualism in predator-prey and competitive systems," Mathematical Biosciences, vol. 65, no. 1, pp. 13-50, 1983.

[2] S. N. Zheng, "A reaction-diffusion system of a competitorcompetitor-mutualist model," Journal of Mathematical Analysis and Applications, vol. 124, no. 1, pp. 254-280, 1987.

[3] S. Xu, "Global stability of a reaction-diffusion system of a competitor-competitor-mutualist model," Taiwanese Journal of Mathematics, vol. 15, no. 4, pp. 1617-1627, 2011.

[4] C. V. Pao, "The global attractor of a competitor-competitormutualist reaction-diffusion system with time delays," Nonlinear Analysis: Theory, Methods \& Applications A, vol. 67, no. 9, pp. 2623-2631, 2007.

[5] W. Chen and R. Peng, "Stationary patterns created by crossdiffusion for the competitor-competitor-mutualist model," Journal of Mathematical Analysis and Applications, vol. 291, no. 2, pp. 550-564, 2004.

[6] M. Li, Z. Lin, and J. Liu, "Coexistence in a competitorcompetitor-mutualist model," Applied Mathematical Modelling, vol. 34, no. 11, pp. 3400-3407, 2010.

[7] S. M. Fu, H. Y. Gao, and S. B. Cui, "Global solutions for the competitor-competitor-mutualist model with cross-diffusion," Acta Mathematica Sinica A, vol. 51, no. 1, pp. 153-164, 2008.

[8] C. Tian and Z. Ling, "Turing pattern formation in a predatorprey-mutualist system," Nonlinear Analysis: Real World Applications, vol. 12, no. 6, pp. 3224-3237, 2011.

[9] A. Tineo, "Asymptotic behavior of solutions of a periodic reaction-diffusion system of a competitor-competitormutualist model," Journal of Differential Equations, vol. 108, no. 2, pp. 326-341, 1994.

[10] Y. Du, "Positive periodic solutions of a competitor-competitormutualist model," Differential and Integral Equations, vol. 9, no. 5, pp. 1043-1066, 1996.

[11] C. V. Pao, "Periodic solutions of parabolic systems with nonlinear boundary conditions," Journal of Mathematical Analysis and Applications, vol. 234, no. 2, pp. 695-716, 1999.

[12] R.-N. Wang, T.-J. Xiao, and J. Liang, "Asymptotic behavior of solutions for systems of periodic reaction-diffusion equations in unbounded domains," International Journal of Evolution Equations, vol. 1, no. 3, pp. 281-298, 2005.

[13] L. Zhou and Y. Fu, "Existence and stability of periodic quasisolutions in nonlinear parabolic systems with discrete delays," Journal of Mathematical Analysis and Applications, vol. 250, no. 1, pp. 139-161, 2000. 
[14] Y. Wang and J. Yin, "Periodic solutions of a class of degenerate parabolic system with delays," Journal of Mathematical Analysis and Applications, vol. 380, no. 1, pp. 57-68, 2011.

[15] K. Gopalsamy, "Exchange of equilibria in two-species LotkaVolterra competition models," Australian Mathematical Society Journal B, vol. 24, no. 2, pp. 160-170, 1982.

[16] C. Alvarez and A. C. Lazer, "An application of topological degree to the periodic competing species problem," Australian Mathematical Society Journal B, vol. 28, no. 2, pp. 202-219, 1986.

[17] S. Ahmad, "Convergence and ultimate bounds of solutions of the nonautonomous Volterra-Lotka competition equations," Journal of Mathematical Analysis and Applications, vol. 127, no. 2, pp. 377-387, 1987.

[18] S. Ahmad and A. C. Lazer, "Asymptotic behaviour of solutions of periodic competition diffusion system," Nonlinear Analysis: Theory, Methods \& Applications A, vol. 13, no. 3, pp. 263-284, 1989.

[19] A. Tineo, "Existence of global coexistence state for periodic competition diffusion systems," Nonlinear Analysis: Theory, Methods \& Applications A, vol. 19, no. 4, pp. 335-344, 1992.

[20] Q. L. Peng and L. S. Chen, "Asymptotic behavior of the nonautonomous two-species Lotka-Volterra competition models," Computers \& Mathematics with Applications, vol. 27, no. 12, pp. 53-60, 1994.

[21] S. Fu and S. Cui, "Persistence in a periodic competitorcompetitor-mutualist diffusion system," Journal of Mathematical Analysis and Applications, vol. 263, no. 1, pp. 234-245, 2001.

[22] W. Dimbour and G. M. N'Guérékata, "S-asymptotically $\omega$ periodic solutions to some classes of partial evolution equations," Applied Mathematics and Computation, vol. 218, no. 14, pp. 7622-7628, 2012.

[23] H. Gao, K. Wang, F. Wei, and X. Ding, "Massera-type theorem and asymptotically periodic logistic equations," Nonlinear Analysis: Real World Applications, vol. 7, no. 5, pp. 1268-1283, 2006.

[24] H. R. Henríquez, "Asymptotically periodic solutions of abstract differential equations," Nonlinear Analysis: Theory, Methods \& Applications A, vol. 80, pp. 135-149, 2013.

[25] J. Wang, M. Feckan, and Y. Zhou, "Nonexistence of periodic solutions and asymptotically periodic solutions for fractional differential equations," Communications in Nonlinear Science and Numerical Simulation, vol. 18, no. 2, pp. 246-256, 2013.

[26] F. Wei and K. Wang, "Asymptotically periodic solution of $N$ species cooperation system with time delay," Nonlinear Analysis: Real World Applications, vol. 7, no. 4, pp. 591-596, 2006.

[27] F. Wei and K. Wang, "Uniform persistence of asymptotically periodic multispecies competition predator-prey systems with Holling III type functional response," Applied Mathematics and Computation, vol. 170, no. 2, pp. 994-998, 2005.

[28] Q. X. Ye and Z. Y. Li, Introduction to Reaction-Diffusion Equations, Science Press, Beijing, China, 1990. 


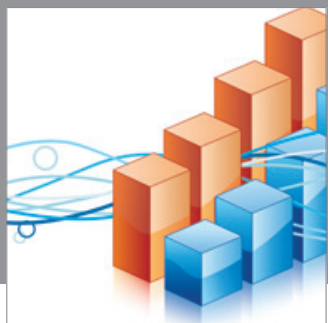

Advances in

Operations Research

mansans

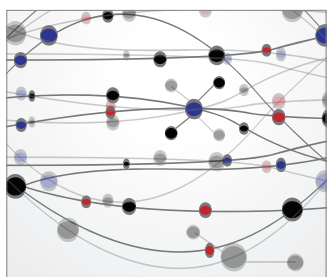

The Scientific World Journal
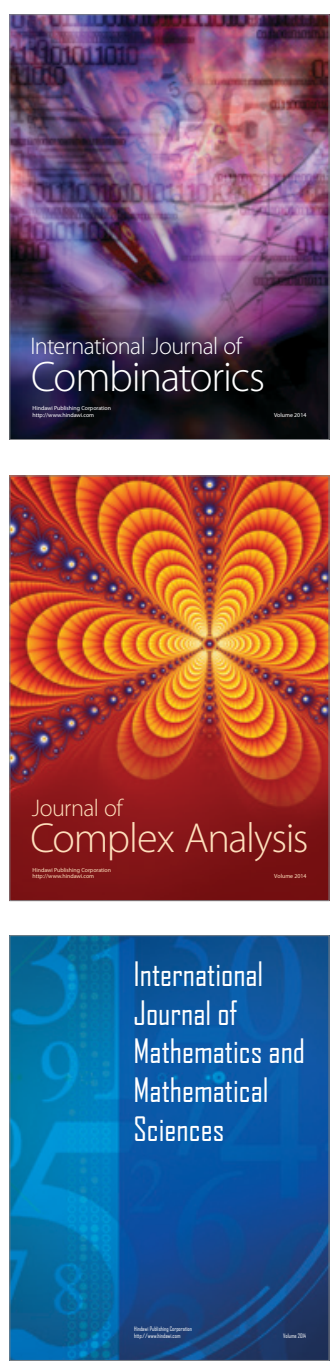
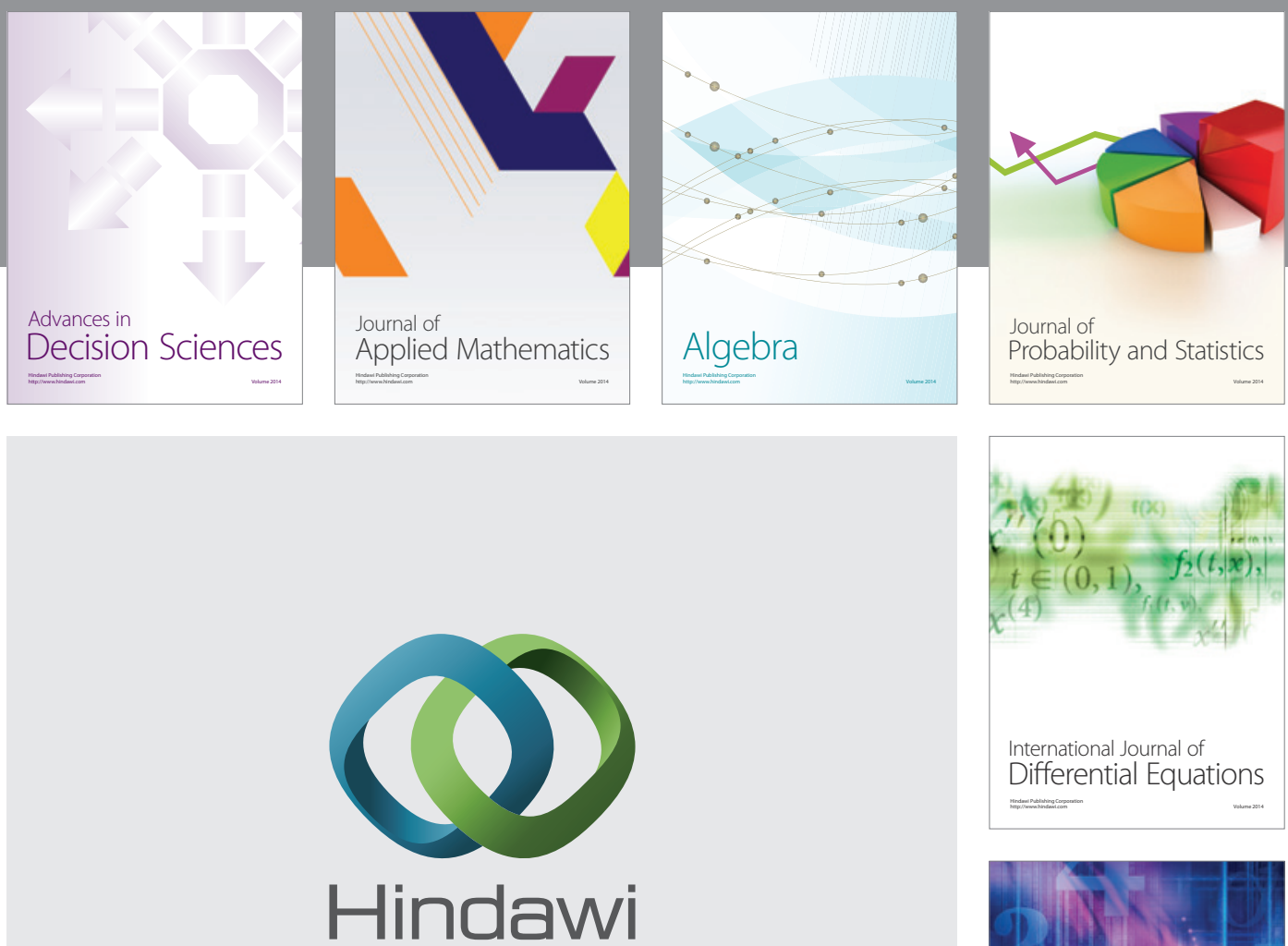

Submit your manuscripts at http://www.hindawi.com
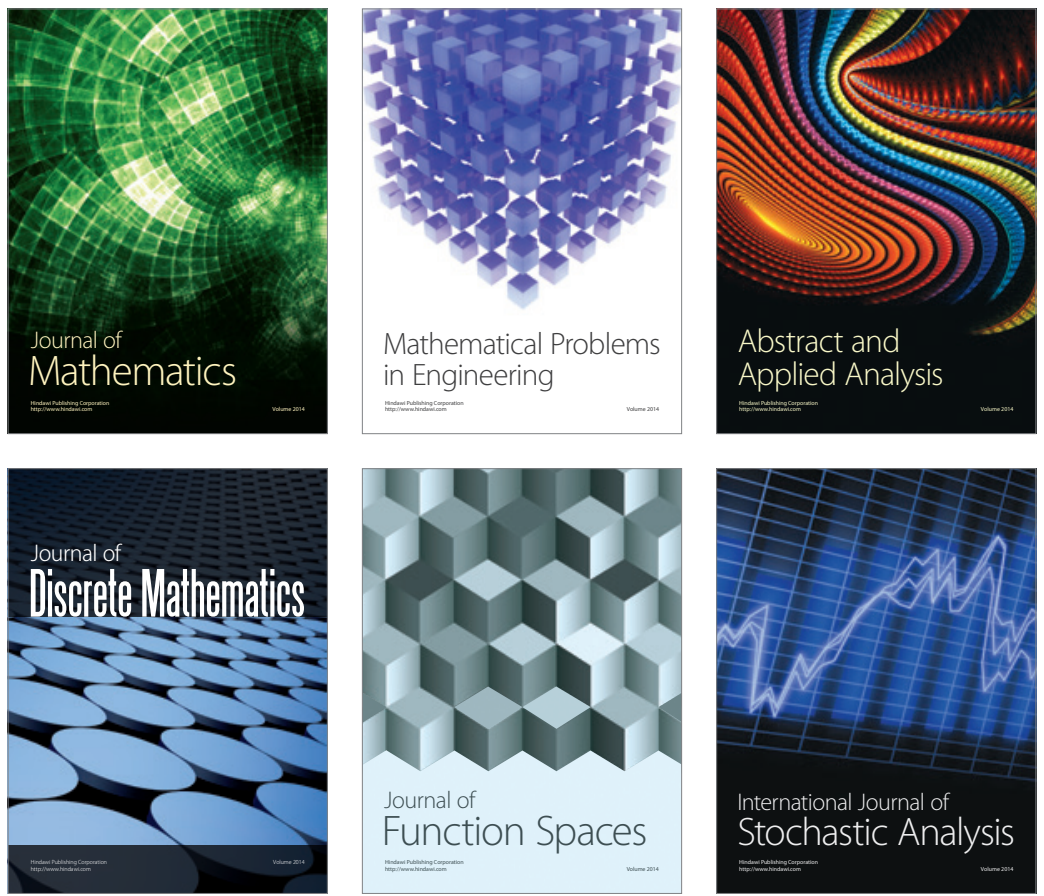

Journal of

Function Spaces

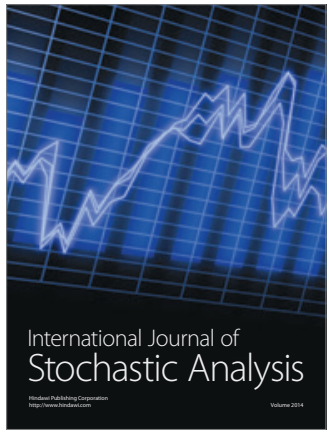

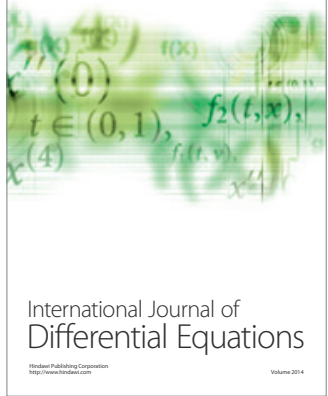
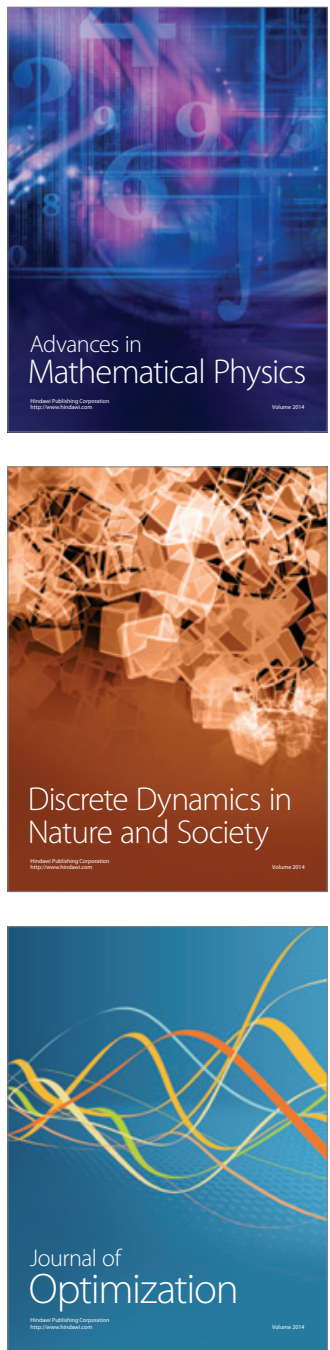